\title{
PJ34, a poly(ADP-ribose) polymerase (PARP) inhibitor, reverses melphalan-resistance and inhibits repair of DNA double-strand breaks by targeting the FA/BRCA pathway in multidrug resistant multiple myeloma cell line RPMI8226/R
}

\author{
TING XIONG, HENG WEI, XIAOQIONG CHEN and HUI XIAO
}

\author{
Department of Hematology, Zhongnan Hospital of Wuhan University, Wuhan, Hubei 430071, P.R. China
}

Received August 19, 2014; Accepted October 3, 2014

DOI: $10.3892 /$ ijo.2014.2726

\begin{abstract}
There is still no ideal treatment for multidrug resistant multiple myeloma, looking for drugs which can reverse chemotherapy resistance and enhance curative effects of chemotherapy drugs becomes a problem that needs to be solved urgently. Poly(ADP-ribose) polymerase inhibitors appear to be an important tool for medical therapy of several malignancies. In the present study, we investigated the potential of the PARP-1 inhibitor PJ34, in vitro, to further enhance the efficacy of the traditional chemotherapy drug melphalan in the multidrug-resistant multiple myeloma cell line RPMI8226/R. The effects of different concentrations of PJ34 and melphalan on cell proliferation were determined by the CCK- 8 assay. The expressions of FA/BRCA pathway-related factors were detected by western blotting and RT-PCR. The percentage of cell apoptosis was measured with flow cytometry. DNA double-strand break (DSB) repair was quantified by $\gamma \mathrm{H} 2 \mathrm{AX}$ immunofluorescence. In addition, DNA damage repair at the level of the individual cell was determined by comet assay. Co-administration of PJ34 and melphalan had synergistic inhibitory effects on the proliferation of RPMI8226/R cells, suggesting more powerful antitumor activities. The apoptosis percentage also was increased more obviously by the treatment of melphalan plus PJ34. The activation of FA/BRCA pathway was inhibited by downregulation of related factors including FANCD2, BRCA2 and Rad51. PJ34 significantly increased the ratio of $\gamma \mathrm{H} 2 \mathrm{AX}$-positive cells and the number of foci/cells. The comet tail rate of cells, tail length, tail moment and Olive tail moment all increased after PJ34 treatment in RPMI8226/R cells. These results indicate that PJ34 combined treatment with melphalan produces synergistic effects and reverses multidrug resistance of RPMI8226/R cells effectively. PJ34 cannot induce DNA damage directly, but it may increase the DNA damage induced by melphalan
\end{abstract}

Correspondence to: Dr Hui Xiao, Department of Hematology, Zhongnan Hospital of Wuhan University, Wuhan, Hubei 430071, P.R. China

E-mail: huixiaowh@hotmail.com

Key words: FA/BRCA (Fanconi anemia/BRCA) pathway, DNA damage repair, multiple myeloma, multidrug resistance through inhibiting DNA damage repair. The suppression of FA/BRCA pathway may be the mechanism. Therefore, we suggest that PARP inhibitors may deserve future investigations as tools for medical treatment of multidrug resistant multiple myeloma.

\section{Introduction}

Multiple myeloma (MM) is a kind of haematological malignancy, which is caused by abnormal proliferation and differentiation of plasma cells in bone marrow. However, there is no ideal treatment for MM, particularly multidrug resistant and refractory MM. As a proteasome inhibitor for biological target therapy, bortezomib has been used to treat MM in recent years. Although bortezomib obtains better curative effect in many MM patients, some patients still relapse after several courses of treatment or they are primary drug resistant to bortezomib. For those patients, combined chemotherapy is indispensable. Multidrug resistance (MDR) of tumor cells towards chemotherapeutic drugs is the main reason for the failure of chemotherapy, so to find selective MDR reversal agents has become important in tumor treatment.

Several investigations have proved that the chemotherapy resistance is related to enhanced DNA damage repair in tumor cells $(1,2)$, but the mechanism of this phenomenon remains ambiguous and open to discussion. Alkylating agents (known as DNA cross-linking agents) are major chemotherapeutic drugs for many hematologic malignancies and solid tumors, such as melphalan and cyclophosphamide are used for the treatment of MM as well as lymphoma and leukemia. Alkylating agents target DNA and induce interstrand cross-link (ICL) damage, thus the cellular replication stops (3). ICLs can cause serious replication forks blocking and DSBs are the result of a stalled replication fork at an ICL $(4,5)$. Studies showed that repair of the ICLs depends upon base excision repair (BER), nucleotide excision repair (NER) and homologous recombination $(\mathrm{HR})(4,6)$.

The Fanconi anemia (FA)/BRCA pathway is a specialized DNA repair pathway that removes interstrand crosslinks (ICLs), a particularly toxic lesion resulting from exposure to alkylating agents such as mitomycin $\mathrm{C}$ and cisplatin (7). The FA/BRCA pathway is initiated when the FA core complex 
(comprised of FANCA/B/C/E/F/G/L/M) recognizes and binds an ICL through an interaction mediated by FANCM and its binding partners (8). Following lesion binding, the FA core complex monoubiquitinates FANCD2 and FANCI via the E3 ubiquitin ligase activity of FANCL. Monoubiquitinated FANCD2/FANCI interact with downstream FA proteins (FANCD1/J/N/O), which form stable complexes with proteins participating in homologous recombination (HR), such as BRCA1 and RAD51 $(9,10)$. The ubiquitinated FANCD2-I heterodimer localizes to the ICL and recruits endonucleases that incise the DNA on either side of the lesion to create a double-stranded break (DSB). The complementary strand containing the unhooked crosslink is replicated by a translesion synthesis (TLS) polymerase, and downstream FA proteins (FANCD1/J/N/O) assist in coordination of HR to repair the DSB (8).

Several studies have proven that the FA/BRCA pathway plays an important role in the process of DNA damage repair (11-13). A variety of alkylating agents exert their effects through the FA/BRCA pathway, so adjusting this pathway may change the effects of the alkylating agents (14-20). Phenylbutyrate had therapeutic utility as a cisplatin sensitizer in head and neck cancer by inhibiting the FA/BRCA pathway through the downregulation of BRCA1, and this sensitization correlated to a significant decrease in the formation of cisplatin-induced FANCD2 nuclear foci, which is a functional read out of the FA/BRCA pathway (21). This abrogation of the FA/BRCA pathway by phenylbutyrate was not due to loss of FANCD2 monoubiquitylation but rather correlated to a phenylbutyrate-mediated reduction in the expression of the BRCA1 protein. In addition, it was found that FANCD1/BRCA2 small interference RNA efficiently enhanced cellular sensitivity toward alkylating agents ACNU and TMZ in human glioblastoma A172 cells (22). Using the proteasome inhibitor bortezomib drastically reduced the FA/BRCA gene expression in myeloma cells, resulting in diminished DNA damage repair and enhanced melphalan sensitivity (23). The above findings suggest that the downregulation of FA/BRCA pathway might be an effective strategy to increase cellular sensitivity toward alkylating agents. These results provide evidence for targeting Fanconi anemia-mediated DNA repair to enhance chemotherapeutic response and circumvent drug resistance in patients with a tumor.

According to related literature, Poly(ADP-ribose) polymerase-1 (PARP-1), as an enzyme critical in BER, plays an important role in DNA repair and perception of DNA damage (24-26). PARP-1 is involved in the repair of singlestrand DNA breaks through the BER pathway and may also be involved in repair of double-strand breaks, through the homologous recombination (HR) pathway (27). Thus, its enzyme activity is regulated by its binding to DNA, recognizing single- and double-strand breaks and also by interacting with a wide variety of chromatin-associated proteins, including components of the transcription machinery, sequence-specific DNA-binding transcription factors, chromatin modifying enzymes and histone variants (28).

PARP-1 inhibition has been shown to block DNA repair, so the inhibitors of PARP-1 are promising assistant drugs in antitumour treatment $(29,30)$. Several studies have confirmed that PARP-1 inhibitors can obviously improve the effects of alkylating agents used in chemotherapy including cisplatin, cyclophosphamide, temozolomide and oxaliplatin (31-34). Enhanced efficacy of PARP-1 inhibitors was most likely caused by disruption of DNA damage repair pathways. In addition, when being applied independently, PARP-1 inhibitors show tumor-selective killing effect (35) and strong inhibition of angiogenesis (36) on tumors with homologous recombination repair defects (such as BRCA1 and BRCA2 defects). However, the role of PARP-1 in DNA damage is not well-understood, and the mechanism of PARP-1 inhibitors increasing the sensitivity of tumor cells to alkylating agents remains to be fully elucidated.

A number of studies show that the FA/BRCA pathway is involved in the repair process of ICL with a variety of components, inhibiting the expression of certain factors in FA/BRCA pathway seems to be capable of suppressing DNA repair and enhancing the sensitivity of tumor cells to alkylating agents (11-23). PARP-1 inhibitors can improve the efficacy of alkylating agents such as cisplatin, and cyclophosphamide due to the inhibition of BER after ICLs caused by alkylating agents (29-34). Thus, we speculate that effects of PARP-1 inhibitors may be associated with inhibition of the FA/BRCA pathway, and the drug resistance to alkylating agent-based chemotherapy might be reversed by inhibiting the activity of this pathway. Therefore, in the present study, we investigated the effect of PJ34, a potent PARP-1 inhibitor, on the melphalan-induced cytotoxicity and DNA damage in a multidrug-resistant cell line RPMI8226/R. We compared the sensitivity of RPMI8226/R cells to melphalan before and after the treatment with PJ34, and detected the expression level of related factors in the FA/BRCA pathway. We attempted to demonstrate the influence of PJ34 on DNA repair and drug resistance and the possible relationship with the FA/BRCA pathway.

\section{Materials and methods}

Materials. The human multiple myeloma cell line RPMI8226 was kindly gifted by Professor Jianfeng Zhou (Department of Hematology, Tongji Hospital of Huazhong University of Science and Technology), the original cell line was purchased from the American Type Culture Collection (ATCC, Manassas, VA, USA). PJ34, melphalan, DMSO were purchased from Sigma Chemical Co. CCK-8 Cell Counting kit was purchased from Dojindo Laboratories (Kumamoto, Japan). RPMI-1640 and fetal calf serum were purchased from Invitrogen Life Technologies. The primary and second antibodies of FANCD2, BRCA2, RAD51, $\gamma \mathrm{H} 2 \mathrm{AX}$ and $\beta$-actin were purchased from Santa Cruz Biotechnology.

Cell culture. RPMI8226 cells were cultured in RPMI-1640 medium supplemented with $10 \%$ fetal calf serum, and incubated in a humidified atmosphere containing of $5 \% \mathrm{CO}_{2}$ at $37^{\circ} \mathrm{C}$. Multidrug-resistant RPMI8226/R cells were cultured in the same environment as above, but they were selected by stepwise exposure of parental sensitive RPMI8226 cells to increasing concentrations of melphalan. The RPMI8226/R cells were maintained in the presence of $4.5 \mu \mathrm{mol} / 1$ melphalan and grown in drug-free medium 2 weeks before the experiments. 
Cytotoxicity and chemosensitivity assay. RPMI8226 and RPMI8226/R cells in the exponential proliferation period were seeded into 96 -well plates ( $1 \times 10^{5}$ cells/well). Twenty-four hours after plating, PJ34, melphalan and melphalan plus PJ34 were added to 3 test groups with increasing drug concentrations. Each concentration was added in quadruplicate. The reversal reagent of the control group was replaced by RMPI1640. The reversal effect of PJ34 was determined by CCK- 8 assay according to the manufacturer's instructions. Drug effects were determined at the level of 50\% inhibition $\left(\mathrm{IC}_{50}\right)$ compared to controls.

Western blot analysis. Cell lysates were prepared by suspending cell pellets in lysis buffer. The proteins were separated by SDS-PAGE and western blot analysis was performed as previously described (37). A total of $50 \mu \mathrm{g}$ protein was used for the western blotting unless otherwise indicated. $\beta$-actin was used as the loading control.

Reverse transcription-polymerase chain reaction (RT-PCR). Total RNA was extracted from RPMI8226/R cells after treatment with PJ34 at different concentrations with TRIzol reagent (Invitrogen, Carlsbad, CA, USA) according to the manufacturer's instruction. cDNA was synthesized using the RevertAid $^{\mathrm{TM}}$ First Strand cDNA Synthesis kit (Fermentas). The final cDNA was used for the subsequent PCR. FANCD2, BRCA2 and RAD51 gene expressions were quantified by semi-quantitative RT-PCR using SYBR-Green/Fluorescein qPCR Master Mix (Fermentas). $\beta$-actin was used as the endogenous control.

Alkaline comet assays. To assess DNA DSBs repair, the comet assay was performed under alkaline conditions using CometSlide assay kits (Trevigen) following the protocol. RPMI8226/R cells were incubated at $37^{\circ} \mathrm{C}$ for $24 \mathrm{~h}$ in three groups (PBS, melphalan or melphalan plus PJ34) to allow for DNA damage repair. Cells were embedded in agarose, lysed, and subjected to alkaline electrophoresis. Immediately before image analysis, cells were stained with ethidium bromide and visualized under a fluorescence microscope. The program CometScore ${ }^{\mathrm{TM}}$ Version 1.5 was utilized to analyze the comet images. The tail length, comet length, tail moment and olive tail moment induced by different treatment were the analysis parameters. A total of 75 cells/sample were scored to determine the average percentage of DNA damaged.

Immunofluorescence microscopy and quantification of $\gamma H 2 A X$ foci. RPMI8226/R cells were incubated at $37^{\circ} \mathrm{C}$ for $24 \mathrm{~h}$ in three groups (PBS, melphalan or melphalan plus PJ34). After treatment, cells were fixed with $4 \%$ paraformaldehyde, permeabilized and blocked by incubation in PBS containing $0.5 \%$ Triton $\mathrm{X}-100,15 \%$ goat serum, $0.2 \%$ fish skin gelatin and $0.03 \% \mathrm{NaN}_{3}$. Next, cells were stained with mouse monoclonal anti- $\gamma \mathrm{H} 2 \mathrm{AX}$ antibody, followed by FITC-conjugated goat antimouse secondary antibody and counterstained with DAPI to visualize the cell nucleus. The percentage of cells containing more than 10 nuclear fluorescent foci per total cell number was calculated by examining a minimum of 120 cells for each experimental group.
Table I. Cross-resistance patterns of RPMI8226 and RPMI8226/R cell lines to various chemotherapeutic agents $(\mu \mathrm{mol} / 1$, mean $\pm \mathrm{SD})$.

\begin{tabular}{lccc}
\hline Agent & $\mathrm{RPMI} 8226\left(\mathrm{IC}_{50}\right)$ & $\mathrm{RPMI} 8226 / \mathrm{R}\left(\mathrm{IC}_{50}\right)$ & $\mathrm{RI}$ \\
\hline Melphalan & $4.79 \pm 0.18$ & $20.43 \pm 0.21$ & 4.27 \\
Ara-C & $1.99 \pm 0.25$ & $6.11 \pm 0.13$ & 3.07 \\
VP-16 & $7.54 \pm 0.08$ & $18.04 \pm 0.12$ & 2.39 \\
DDP & $1.30 \pm 0.03$ & $5.55 \pm 0.01$ & 4.26 \\
ADM & $0.73 \pm 0.16$ & $3.07 \pm 0.32$ & 4.20 \\
CTX & $2.89 \pm 0.09$ & $25.14 \pm 0.02$ & 8.70 \\
VCR & $1.63 \pm 0.30$ & $4.41 \pm 0.41$ & 2.71 \\
\hline
\end{tabular}

Apoptosis assay. As previously described (37), to detect apoptosis, cells of the four groups were washed in PBS, resuspended in $500 \mu \mathrm{l}$ binding buffer containing Annexin V-FITC/PI and analyzed by flow cytometry.

Statistical analysis. SPSS 13.0 was used to perform the statistical analysis. Data are presented as mean $\pm \mathrm{SD}$, and analyzed by the Student's t-test. $\mathrm{P}<0.05$ was considered statistically significant.

\section{Results}

Establishment and characterization of the RPMI8226/R cells. The multidrug resistant RPMI8226/R cell line was established with a $4.5 \mu \mathrm{mol} / 1$ final concentration of melphalan. In order to identify the establishment of resistance to different anticancer agents, the sensitivities of the RPMI8226/R and the RPMI8226 cells were compared. According to the CCK-8 assay results, the RPMI8226/R cells exhibited resistance not only to melphalan, but also to six other anticancer agents: adriamycin (ADM), cyclophosphamide (CTX), cisplatin (DDP), Ara-C, vincristine (VCR) and VP-16. The relative resistance of RPMI8226/R cells, as compared to the RPMI8226, is demonstrated in Table I. The $\mathrm{IC}_{50}$ values for RPMI8226/R and RPMI8226 were $20.43 \pm 0.21$ and $4.79 \pm 0.18 \mu \mathrm{mol} / 1$, respectively. The degree of resistance was evaluated in terms of resistance index (RI) which is calculated according to the relation: $\mathrm{RI}=\mathrm{IC}_{\text {50(RPMI8226/R) }} / \mathrm{IC}_{50(\mathrm{RPMI} I 8226)}$. Therefore, RPMI8226/R cell line was $\sim 4.27$-fold more resistant to melphalan than the original cell line. Moreover, RPMI8226/R cell line was 8.70-fold more resistant to CTX, another alkylating agent. When RPMI8226/R cell line was cultured in RPMI-1640 without melphalan for 10 weeks, there was no drug resistance decrease with the similar RI as before $(R I=4.10)$, showing the cell line had stable drug resistance, but the RI decreased to 3.05 after 12 weeks and recovered to 4.21 by adding melphalan $(4.5 \mu \mathrm{M})$ into the medium again.

PJ34 enhanced the toxicity of melphalan in RPMI8226/R cells but not in RPMI8226 cells. In the present study, we investigated the effect of PJ34 combined with melphalan on the viability of RPMI8226 and RPMI8226/R cells by CCK-8 assay. As shown in Fig. 1A and Table IIA, PJ34 alone did not exhibit a cytotoxic effect on RPMI8226/R cells at doses up to $60 \mu \mathrm{M}$. 
Table II. The effects of PJ34 on cell growth in RPMI8226 and RPMI8226/R.

A, The effects of PJ34 on cell growth of RPMI8226 and RPMI8226/R

\begin{tabular}{lrrr}
\hline & \multicolumn{3}{c}{ Cell growth $(\%)$} \\
\cline { 2 - 4 } PJ-34 $(\mu \mathrm{M})$ & RPMI8226 & RPMI8226/R & P-value \\
\hline 3 & $88.64 \pm 2.00$ & $95.90 \pm 2.62$ & 0.055 \\
10 & $84.93 \pm 1.00$ & $87.02 \pm 1.08$ & 0.068 \\
30 & $55.95 \pm 1.94$ & $67.80 \pm 1.51$ & 0.017 \\
60 & $45.57 \pm 1.51$ & $48.95 \pm 1.22$ & 0.109 \\
\hline
\end{tabular}

B, The effects of PJ34 on RPMI8226/R growth inhibited by melphalan

\begin{tabular}{lccr}
\hline & \multicolumn{2}{c}{ Cell growth $(\%)$} \\
\cline { 2 - 4 } Melphalan $(\mu \mathrm{M})$ & RPMI8226/R & RPMI8226/R+PJ34 & P-value \\
\hline 4.8 & $89.47 \pm 0.50$ & $69.92 \pm 0.33$ & 0.000 \\
9.6 & $80.19 \pm 0.45$ & $41.81 \pm 0.13$ & 0.000 \\
12.8 & $70.41 \pm 0.50$ & $31.87 \pm 0.22$ & 0.000 \\
16 & $58.42 \pm 0.47$ & $18.13 \pm 0.28$ & 0.000 \\
\hline
\end{tabular}

C, The effects of PJ34 on RPMI8226 growth inhibited by melphalan

Cell growth $(\%)$

\begin{tabular}{lccc}
\cline { 2 - 3 } Melphalan $(\mu \mathrm{M})$ & RPMI8226 & RPMI8226+PJ34 & P-value \\
\hline 4.8 & $50.08 \pm 1.33$ & $48.56 \pm 1.10$ & 0.28 \\
9.6 & $34.41 \pm 2.05$ & $28.65 \pm 2.30$ & 0.053 \\
12.8 & $26.12 \pm 2.50$ & $21.57 \pm 2.02$ & 0.061 \\
16 & $14.84 \pm 0.75$ & $14.24 \pm 1.40$ & 0.094 \\
\hline
\end{tabular}

To test synergistic antitumor effect of PJ34 and melphalan in RPMI8226/R cells, the RPMI8226/R cells were exposed to increasing doses of PJ34 alone, melphalan alone or melphalan in combination with $60 \mu \mathrm{M}$ of PJ34 and antitumor effect was assessed by CCK-8 assay (Fig. 1). For RPMI8226/R cells, the $\mathrm{IC}_{50}$ value of PJ34 was $60 \mu \mathrm{M}$, melphalan was $20.43 \mu \mathrm{M}$, and the combination $7.8 \mu \mathrm{M}$ (Table II).

Compared with DMSO-pretreated cells, pretreatment with $60 \mu \mathrm{M}$ PJ34 resulted in significant inhibition of cell growth in RPMI8226/R cells after melphalan treatment (Fig. 1B, Table IIB). Similar result was not observed in RPMI8226 cells (Fig. 1C, Table IIC). Taken together, the pretreatment of PJ34 at $60 \mu \mathrm{M}$ consistently enhanced the toxicity of melphalan in RPMI8226/R cells, but not in RPMI8226 cells. In RPMI8226/R cells, $\mathrm{IC}_{50}$ of melphalan with $60 \mu \mathrm{M}$ of PJ34 reduced from 20.43 to $7.82 \mu \mathrm{M}$. Therefore, $60 \mu \mathrm{M}$ of PJ34 was selected for further experiments. The use of PJ34 combined with melphalan led to a more significant reduction in viability (increased toxicity) than the use of either PJ34 or melphalan alone in RPMI8226/ $\mathrm{R}$ cells $(\mathrm{P}<0.05)$. These results all clearly indicate that PJ34 is a potential chemosensitizer of melphalan.

PJ34 suppressed the $m R N A$ and protein expressions of the factors in FA/BRCA pathway in RPMI8226/R cells. The two cell lines, RPMI8226/R and RPMI8226, were exposed to various concentrations of PJ34 for $24 \mathrm{~h}$. The protein expression of FANCD2, BRCA2 and Rad51 in RPMI8226/R cells was higher than that in RPMI8226 cells (Fig. 2). In contrast, the expression of $\gamma \mathrm{H} 2 \mathrm{AX}$ in RPMI8226/R cells was lower than that in RPMI8226 cells before PJ34 treatment. It was found that PJ34 $(60 \mu \mathrm{M})$ treatment decreased the protein levels of FANCD2, BRCA2, Rad51 and the effect was the opposite on $\gamma \mathrm{H} 2 \mathrm{AX}$ expression in RPMI8226/R cells (Fig. 2; $\mathrm{P}<0.05)$. To elucidate whether the observed effects of PJ34 on the above protein expression occurred at the transcriptional level, total RNA was isolated and subjected to RT-PCR analysis for FANCD2, BRCA2 and Rad51 transcripts. Exposure to PJ34 decreased FANCD2, BRCA2 and Rad51 mRNA levels dose-dependently (Fig. 3). The above results showed that the activity of FA/BRCA pathway was enhanced in the resistant cell line RPMI8226/R, which resulted in intensive repair to the DNA damage induced by melphalan. Whereas the DNA damage of $\gamma \mathrm{H} 2 \mathrm{AX}$ protein expression 

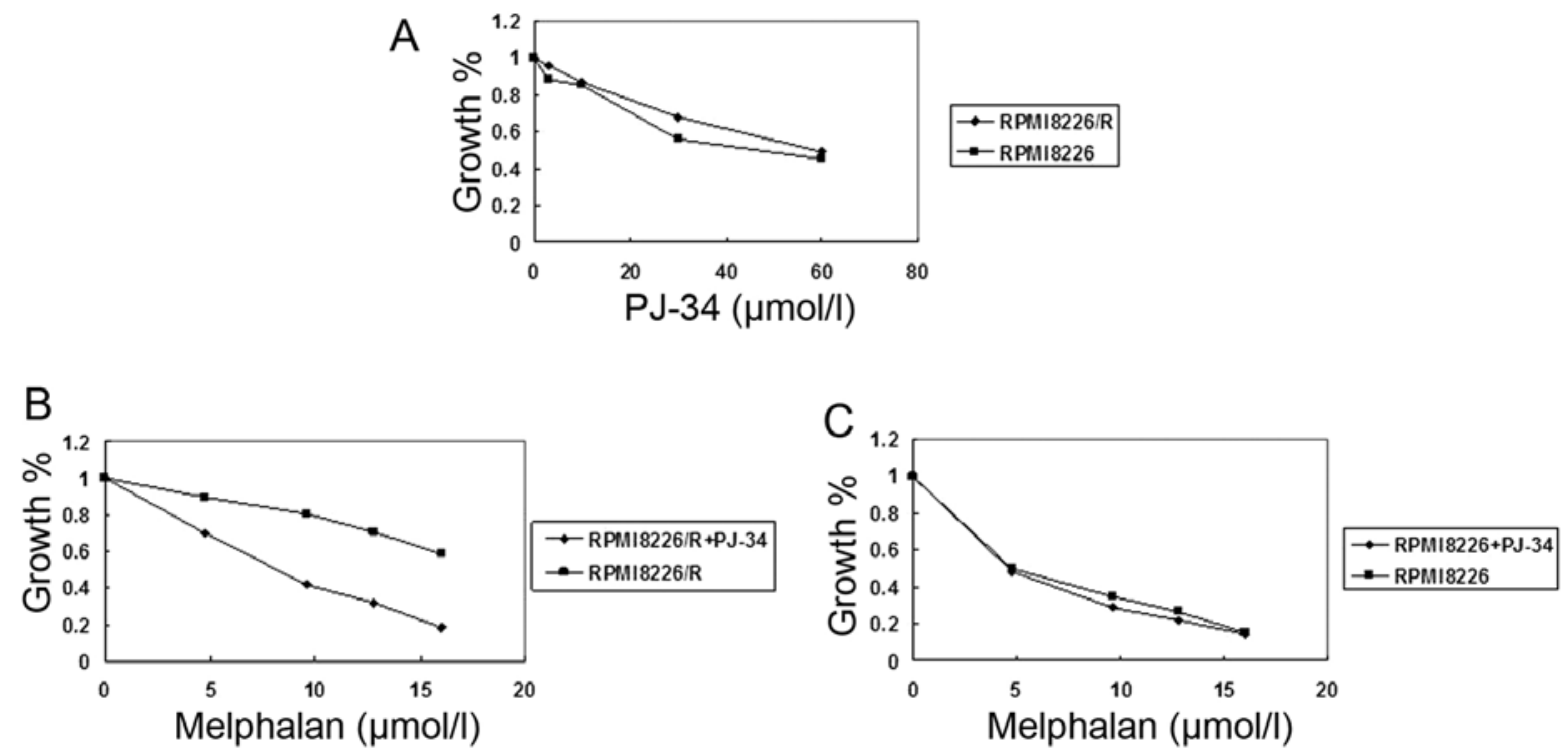

Figure 1. The growth curves for the response of RPMI8226/R cells to melphalan and/or PJ34 as compared to response of RPMI8226 cells. (A) CCK-8 assay comparing RPMI8226/R cells with parental RPMI8226 cells. IC $_{50}$ values of PJ34 were $60 \mu \mathrm{M}$ in both cell lines. (B and C) The effects of melphalan on cell proliferation in the presence of PJ34. Each cell line was treated with 4 concentrations of melphalan $(4.8,9.6,12.8$ and $16 \mu \mathrm{M})$ plus PJ34 (60 $\mu \mathrm{M})$ or not. Cells were cultured for $24 \mathrm{~h}$ and the cell proliferation was evaluated by CCK- 8 assay. All experiments were repeated 3 times, and each value is shown as the mean of 3 experiments \pm SD. (B) Comparative analysis of the antiproliferative effect of melphalan with or without $60 \mu \mathrm{M}$ of PJ34 on RPMI8226/R cells. Synergistic antitumor effect of melphalan and PJ34 was found in RPMI8226/R cells, $\mathrm{IC}_{50}$ of melphalan reduced from 20.43 to $7.82 \mu \mathrm{M}$ when combined with $60 \mu \mathrm{M}$ of PJ34. (C) Comparative analysis of the antiproliferative effect of melphalan with or without $60 \mu \mathrm{M}$ of PJ34 on RPMI8226 cells. Similar synergistic antitumor effect of melphalan and PJ34 was not found in RPMI8226 cells, the two $\mathrm{IC}_{50}$ values were almost the same.
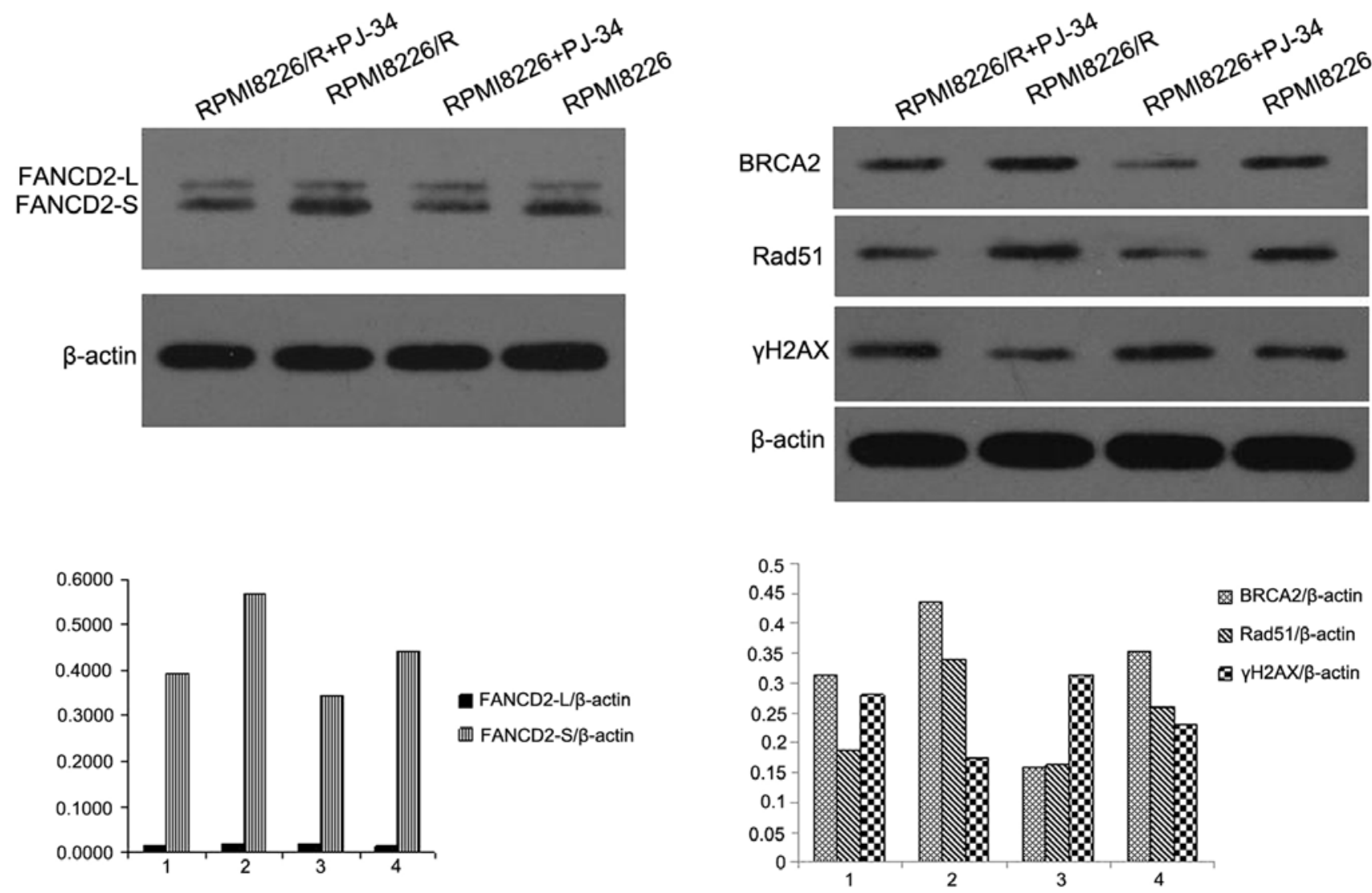

Figure 2. The effects of PJ34 on the FA/BRCA pathway related proteins expression in RPMI8226 and RPMI8226/R cells. PJ34 decreased the protein expression levels of FANCD2, BRCA2 and Rad51 after treatment with $60 \mu \mathrm{M}$ of PJ34 for $24 \mathrm{~h}$ (lane 1, after the treatment of PJ34 in RPMI8226/R; lane 2, before the treatment of PJ34 in RPMI8226/R; lane 3, after the treatment of PJ34 in RPMI8226; lane 4, before the treatment of PJ34 in RPMI8226). Western blot assay showed that higher basal expression levels of FANCD2, BRCA2 and Rad51 in RPMI8226/R cells compared with those in RPMI8226 cells, treatment with $60 \mu \mathrm{M}$ of PJ34 for $24 \mathrm{~h}$ led to downregulation of FANCD2, BRCA2 and Rad51 ( $<<0.05)$ in RPMI8226/R cells compared with those before the treatment with PJ34 in RPMI8226/R cells. 

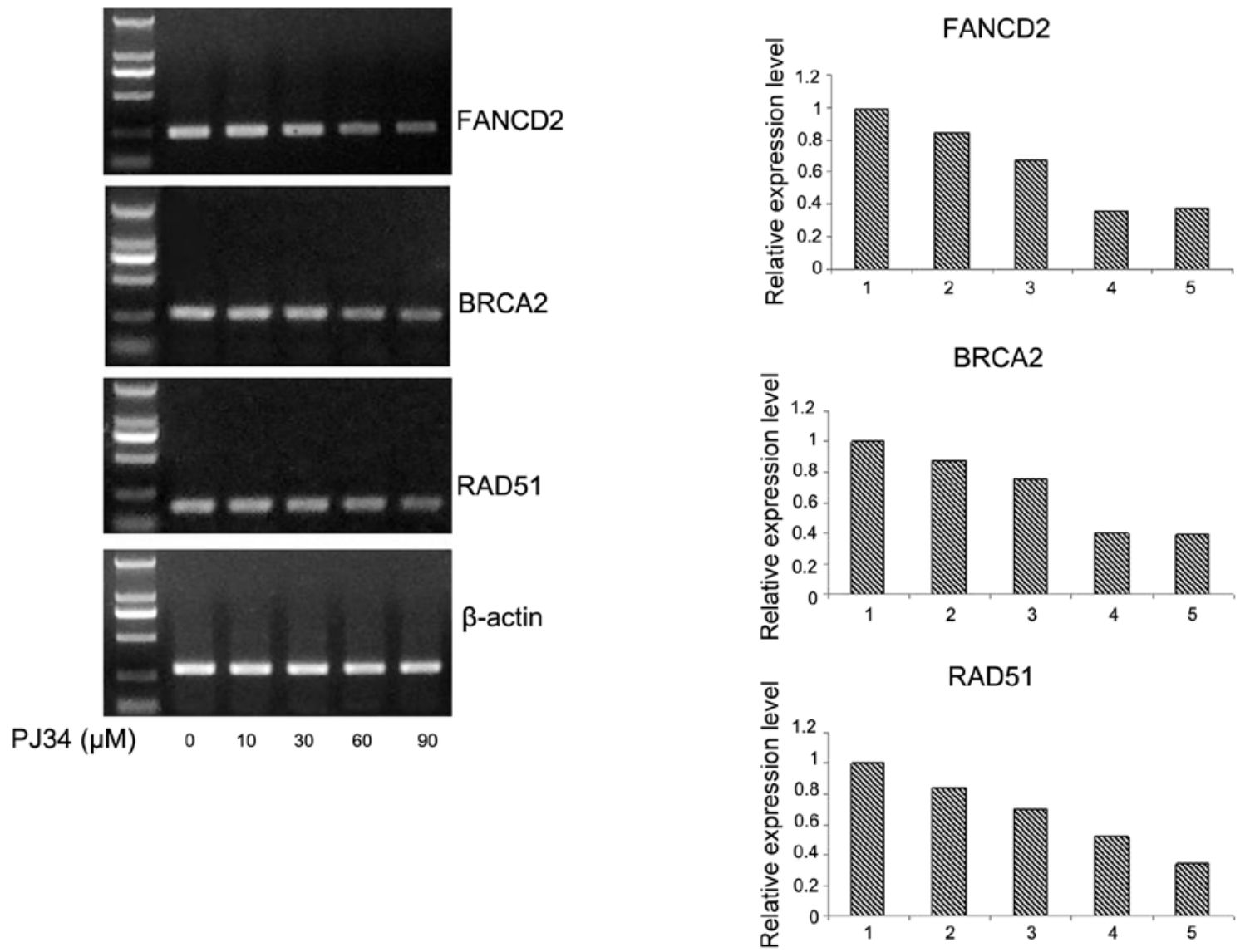

Figure 3. The effects of PJ34 treatment at different concentrations on mRNA expression of the FA/BRCA pathway-related factors in RPMI8226/R cells. Relative expression level: an mRNA//-actin mRNA (lane 1, PJ34 $0 \mu \mathrm{M}$; lane 2, PJ34 $10 \mu \mathrm{M}$; lane 3, PJ34 $30 \mu \mathrm{M}$; lane 4, PJ34 $60 \mu \mathrm{M}$; lane 5, PJ34 $90 \mu \mathrm{M}$ ).

Table III. The effects of PJ34 on cell apoptosis induced by melphalan.

\begin{tabular}{lccc}
\hline & \multicolumn{2}{c}{ Cell apoptosis (\%) } & \\
\cline { 2 - 3 } Group & RPMI8226 & RPMI8226/R & P-value \\
\hline Control & $15.28 \pm 2.84$ & $6.73 \pm 0.28$ & 0.144 \\
PJ-34 & $22.70 \pm 2.16$ & $17.82 \pm 1.12$ & 0.105 \\
Melphalan & $31.08 \pm 0.47$ & $23.88 \pm 1.38$ & 0.02 \\
PJ-34+ melphalan & $45.38 \pm 4.53$ & $55.68 \pm 0.78$ & 0.003 \\
\hline
\end{tabular}

RPMI8226/R and RPMI8226 cells were treated with $9.6 \mu \mathrm{M}$ of melphalan, PJ34 of $60 \mu \mathrm{M}$ alone or in combination for $24 \mathrm{~h}$, and induction of apoptosis was assessed by Annexin V-FITC/ PI flow cytometry. Experiments were performed in triplicate. For RPMI8226/R cell line, neither PJ34 $(60 \mu \mathrm{M})$ nor melphalan $(9.6 \mu \mathrm{M})$ single-agent treatment caused any significant changes in the percentage of cell apoptosis, while the concomitant treatment resulted in a marked increase of cell apoptosis.

declined. The antagonism effects of PJ34 led to suppressed mRNA and protein expressions of the factors in the FA/ BRCA pathway and enhanced DNA damage caused by melphalan in RPMI8226/R cells. In summary, inhibition of the FA/BRCA pathway activity by PJ34 could reduce DNA

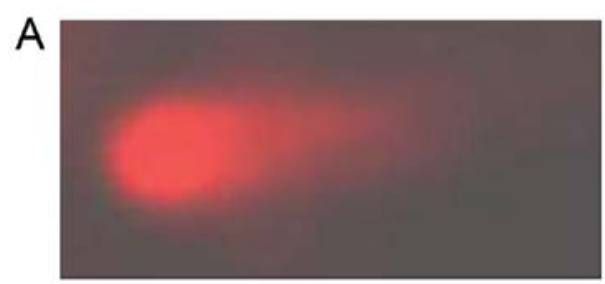

Melphalan+PJ34

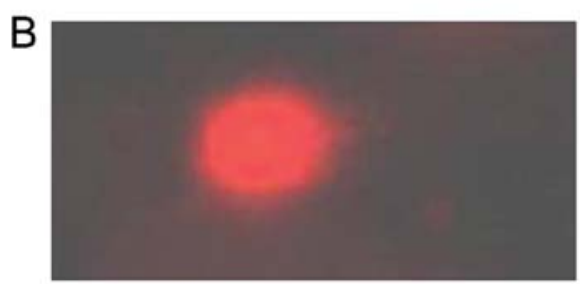

Melphalan

Figure 4. Comet assay images of RPMI8226/R cells. (A) RPMI8226/R cells exposed to melphalan for $24 \mathrm{~h}$ followed by $24-\mathrm{h}$ incubation with PJ34 before comet assay; (B) RPMI8226/R cells exposed to melphalan for $24 \mathrm{~h}$ followed by $24-\mathrm{h}$ incubation without PJ34 before the comet assay.

repair to reverse the drug resistance in melphalan-exposed RPMI8226/R cells.

PJ34 plus melphalan enhance cell apoposis in RPMI8226/R cells. To investigate the effects of PJ34 on cell apoptosis in 
Table IV. The effects of PJ34 on DNA damage in RPMI8226/R cells examined by the comet assay.

\begin{tabular}{lccccc}
\hline Group & Tail length & Comet length & Tail moment & Olive tail moment & Comet-like cell (\%) \\
\hline RPMI8226/R & $12.97 \pm 3.31$ & $40.81 \pm 8.97$ & $1.53 \pm 0.40$ & $1.89 \pm 0.37$ & $20.27 \pm 2.22$ \\
RPMI8226/R+PJ-34 & $31.08 \pm 10.13$ & $66.39 \pm 12.81$ & $7.58 \pm 4.70$ & $6.35 \pm 3.02$ & $45.38 \pm 2.91$ \\
P-value & 0.01 & 0.014 & 0.027 & 0.018 & 0.003 \\
\hline
\end{tabular}

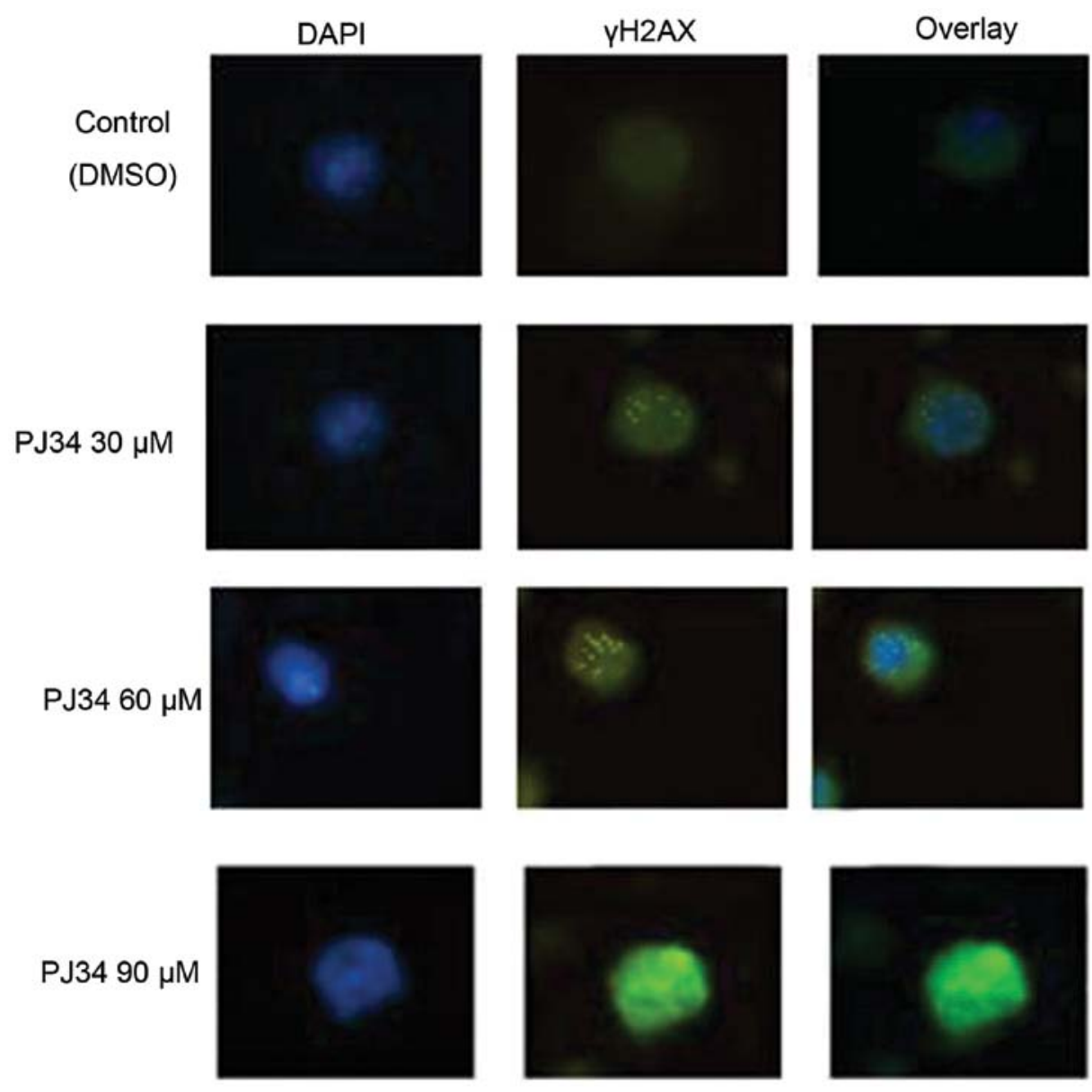

Figure 5. PJ34 induced $\gamma \mathrm{H} 2 \mathrm{AX}$ foci formation in RPMI8226/R cells. After treatment with PJ34 for $24 \mathrm{~h}$, cells were fixed and stained with anti- $\gamma \mathrm{H} 2 \mathrm{AX}$ antibody, and subjected to immunofluorescent microscopy. DAPI: 4',6-diamidino-2-phenylindole. Shown are representative images from one of four independent experiments. (A) DMSO group; (B) PJ34 $30 \mu \mathrm{M}$ treated group; (C) PJ34 $60 \mu \mathrm{M}$ treated group; (D) PJ34 $90 \mu \mathrm{M}$ treated group. Blue, DAPI stain for nuclei; green, $\gamma \mathrm{H} 2 \mathrm{AX}$ foci. Overlay: DAPI stain and $\gamma \mathrm{H} 2 \mathrm{AX}$ foci overlapped. The experiments were repeated 3 times, and each value is shown as the mean of 3 experiments \pm SD.

RPMI8226/R cells treated with melphalan, cells were given differential treatment followed by flow cytometric analysis. As shown in Table III, when cells were treated with melphalan or PJ34 alone, the apoptosis percentage of RPMI8226 was significantly higher than that of RPMI8226/R $(\mathrm{P}<0.05)$. On the contrary, the apoptosis percentage of RPMI8226/R cells was significantly higher than that of RPMI8226 $(\mathrm{P}<0.01)$ with the treatment of melphalan $9.6 \mu \mathrm{M}$ plus PJ34 $60 \mu \mathrm{M}$. Table III also showed significantly increased population of apoptotic cells in concomitant treatment group (55.68 $\pm 0.78 \%)$ compared with untreated control $(6.73 \pm 0.28 \%)$ and melphalan $(23.88 \pm 1.38 \%)$ or PJ34 $(17.82 \pm 1.12 \%)$ single treatment groups $(\mathrm{P}<0.05)$. Therefore, $\mathrm{PJ} 34$ improved the apoptosis percentage of RPMI8226/R cells significantly.
PJ34 increases DNA strand breaks induced by melphalan in RPMI8226/R cells. Treatment with melphalan led to the breakage of DNA strands. Following unwinding, DNA fragments left the nuclear zone and moved to the positive pole under the effect of the electric field in the electrophoresis liquid, forming the distinctive comet tail formation. Thus, damage to cellular DNA of the RPMI8226/R cells due to exposure to melphalan combined with PJ34 or without PJ34 were revealed by alkaline comet assay (Fig. 4). A manifest difference of chemosensitivity to melphalan was observed in RPMI8226/R cells following exposure to PJ34 at $60 \mu \mathrm{M}$, as shown in Table IV. The differences of tail length, comet length, tail moment, olive tail and percentage of comet-like cell between PJ34 treated group and untreated group were all 
Table V. Quantification of $\gamma \mathrm{H} 2 \mathrm{AX}$ foci formation in RPMI8226/R cells.

Group

$\gamma \mathrm{H} 2 \mathrm{AX}$ positive cells $(\%)$

1. Melphalan+DMSO

$15.1 \pm 1.4$

2. Melphalan+PJ34 $30 \mu \mathrm{M}$

$26.5 \pm 3.3$

3. Melphalan+PJ34 $60 \mu \mathrm{M}$

$53.4 \pm 5.6$

4. Melphalan $+\mathrm{PJ} 3490 \mu \mathrm{M}$

$64.3 \pm 6.4$

Group 2 vs. Group 1, P=0.016; Group 2. vs. Group 3, P=0.019; significant differences were found between any other two groups $(\mathrm{P}=0.000)$.

significant $(\mathrm{P}<0.05)$ (Table IV). Increased DNA damage of RPMI8226/R cells with PJ34 treatment was detected. The data revealed that $\sim 80 \%$ of cells did not exhibit any DNA damage in PJ34 untreated group compared to 55\% in PJ34 treated group. The data also showed a significant increase $(\mathrm{P}=0.018)$ in Olive tail moment for PJ34 treated group compared to the control one, and the length of comet tail was 2.40 -fold increased. The results suggested that the DNA damage might be corrected by natural repair mechanisms after incubation without PJ34 and PJ34 was able to enhance DNA damage induced by melphalan in RPMI8226/ $\mathrm{R}$ cells.

PJ34 induces the formation of $\gamma H 2 A X$ foci and blocks the repair of melphalan-induced DNA damage in RPMI8226/R cells. Among the many factors that may affect cell sensitivity to DNA damage, DNA repair is the most critical one. Histone $\mathrm{H} 2 \mathrm{AX}$ phosphorylation $(\gamma \mathrm{H} 2 \mathrm{AX})$ forms nuclear foci that can be visualized by immunostaining using anti- $\gamma \mathrm{H} 2 \mathrm{AX}$ antibody. These foci correspond 1:1 with unrepaired DSBs. So the presence of $\gamma \mathrm{H} 2 \mathrm{AX}$ nuclear foci is regarded as a sensitive indicator for the presence of DSB in chromosomal DNA, and the number of $\gamma \mathrm{H} 2 \mathrm{AX}$ foci is believed to be closely related to the number of DSB in the cells. To assess the role of PJ34 in DSB repair, we used immunofluorescence analysis and quantification of $\gamma \mathrm{H} 2 \mathrm{AX}$ positive RPMI8226/R cells after exposure to various concentrations of PJ34 combined with melphalan $(9.6 \mu \mathrm{M})$ for $24 \mathrm{~h}$. It was found that although majority of the control DMSOtreated cells had no $\gamma \mathrm{H} 2 \mathrm{AX}$ foci in the nuclei, there were still around $15 \%$ of cells containing more than 10 foci. On the other hand, various concentrations of PJ34 all induced increases in the percentage of $\gamma \mathrm{H} 2 \mathrm{AX}$ positive cells (Table $\mathrm{V}$ ). As shown in Fig. 5 and Table $\mathrm{V}$, the percentage of $\gamma \mathrm{H} 2 \mathrm{AX}$ positive cells increased significantly after exposure to increased concentrations of PJ34 combined with melphalan $(\mathrm{P}<0.05)$, indicating that there is more DNA damage. The percentage of $\gamma \mathrm{H} 2 \mathrm{AX}$ positive cells decreased in control group with melphalan treatment alone revealed that DSBs were repaired with time, while there was significantly higher number of residual $\gamma \mathrm{H} 2 \mathrm{AX}$ foci in RPMI8226/R cells when PJ34 was added with melphalan (Table V). PJ34 alone did not induce $\gamma \mathrm{H} 2 \mathrm{AX}$ (data not shown). To further confirm the role of PJ34 in DSB repair, we also evaluated the effects of PJ34 on $\gamma \mathrm{H} 2 \mathrm{AX}$ protein levels after melphalan treatment in RPMI8226/R cells by western blotting. As shown in Fig. 2, after exposure to $60 \mu \mathrm{M}$ of PJ34, the
RPMI8226/R and RPMI8226 cells both retained higher levels of $\gamma \mathrm{H} 2 \mathrm{AX}$ than controls. Moreover, after exposure to $90 \mu \mathrm{M}$ of PJ34, a sub-population of cells even displayed high levels of uniform pan-nuclear $\gamma \mathrm{H} 2 \mathrm{AX}$ staining without identifiable $\gamma \mathrm{H} 2 \mathrm{AX}$ foci (Fig. 2). These data strongly supported that PJ34 slowed down the repair of DSB.

Our results suggested that the effect of melphalan on initial DNA damage was enhanced by PJ34 in RPMI8226/R cells. Despite the more rapid removal of the DSB, more DSBs remained in RPMI8226/R cells due to the excessive level of initial DSB. The net outcome was that RPMI8226/R cells consistently bear more DNA damage after 24-h treatment with melphalan combined with PJ34, which likely contributed to the increased cellular sensitivity to melphalan.

Taken together, all the above results indicated that the PARP-1 inhibitor PJ34 increased melphalan-induced activation of the FA/BRCA pathway and caused persistent melphalaninduced DNA damage.

\section{Discussion}

MM accounts for $\sim 10 \%$ of hematologic malignant tumors. At first MM patients respond well to alkylating agent-based chemotherapy regimens, but almost all patients will relapse after achieving a degree of remission, even those with complete remission (CR). Repeated chemotherapies are more likely to cause multidrug resistance, MDR will result in refractory multiple myeloma. In recent years, although the targeted therapeutic drugs (thalidomide, lenalidomide and bortezomib) have made certain curative effects in cancer clinical treatment, there are still some patients who relapse or die within a year after therapy. Currently autologous hematopoietic stem cell transplantation (Auto-HSCT) can improve survival rate in patients with MM, but most of the patients due to old age are not suitable for Auto-HSCT. Therefore, looking for more effective chemotherapy drugs, improving the curative effects of advanced stage patients, is still the topic needing further exploration.

The alkylating agent-based chemotherapy regimens play important roles in treatment of MM, multidrug resistance is the main impact factor for the survival of patients with MM. Research has suggested that the mechanism of chemotherapy drug resistance mainly includes: increased drug efflux, changes in drug resistance related genes, enhanced DNA damage repair and suppressed apoptosis $(38,39)$. Among them, DNA damage repair is a hotspot of research on the alkylating agent resistance mechanism and MM individualized treatment. The FA/BRCA pathway plays an important role in the regulation of DNA damage repair, it can adjust intracellular reactions to DDP and other alkylating agents (14-23). We speculate that the FA/BRCA pathway may be associated with the chemotherapy drug resistance. Recent research of MDR is focused on searching for potential targets for chemical sensitization agents. Many agents have been confirmed as inhibitors of MDR, but the effective one without toxic side effects has not been found yet. Thus, we need to continue seeking for MDR inhibitors with low toxicity. PARP-1 inhibitor PJ34 is such a candidate drug, its antitumor activity is known, and it can reverse drug resistance by infiltration and enhanced gathering the target cells $(35,36,40-42)$. 
As in our previous study (37), we proposed that the activation of FA/BRCA pathway might contribute to enhanced DNA ICL repair capacity in drug-resistant cells, this pathway should contribute to the acquired drug resistance of tumor cells to DNA crosslinking agents, so the FA/BRCA pathway represents a new target for preventing acquired drug resistance. It is our intention to further enhance the effectiveness of chemotherapy in multidrug-resistant MM patients by identifying other relevant drugs that synergize with alkylating agents. Based on previous reports that the PARP-1 inhibitors can exert synergism with various conventional chemotherapeutic agents including cisplatin, cyclophosphamide, temozolomide and oxaliplatin in tumors (31-34), we reasoned that the PARP-1 inhibitor PJ34 in conjunction with melphalan, might enhance antitumor effect of both agents in multidrug resistant MM cells. To test this hypothesis, we performed a synergy test for the PJ34 and melphalan and found a significant enhancement in the antitumor effect compared with that of either agent as a single treatment. Our results demonstrated that when used in combination to treat multidrug resistant RPMI8226/R cells, the $\mathrm{IC}_{50}$ of melphalan could be reduced by 2.6 -fold. Flow cytometry also showed that combination treatment of PJ34 and melphalan caused a marked increase in apoptosis. Western blot analysis and RT-PCR demonstrated that the exposure of RPMI8226/ $\mathrm{R}$ cells to combined treatment induced a marked decrease in FANCD2, BRCA2 and Rad51 protein and mRNA expression levels, which causing downregulation of the activity of the FA/BRCA pathway, suggesting the suppression of this signaling pathway by PJ34 and melphalan co-treatment. Moreover, significant differences in DNA damage repair were observed between treatment with melphalan alone and treatment with melphalan and PJ34 in RPMI8226/R cells, as observed using the comet assay and $\gamma \mathrm{H} 2 \mathrm{AX}$ foci analysis, which demonstrated that the degree of DNA damage caused by melphalan. Histone $\mathrm{H} 2 \mathrm{AX}$ is the sensor of DNA damage, one of the early occurred events after DNA DSBs is the formation of phosphorylated histone $\mathrm{H} 2 \mathrm{AX}(\gamma \mathrm{H} 2 \mathrm{AX}), \gamma \mathrm{H} 2 \mathrm{AX}$ is the gold standard for detecting DSB (43). Therefore, the level of $\gamma \mathrm{H} 2 \mathrm{AX}$ can reflect the DNA damage caused by chemotherapy drugs in tumor cells, and also can be the index of chemotherapy sensitivity. The present study also found that the protein expression level of $\gamma \mathrm{H} 2 \mathrm{AX}$ increased after exposure to PJ34 in RPMI8226/R cells, indicating increasing DSBs and enhanced drug sensitivity. Our results demonstrate for the first time that PJ34 can decrease the expression of the factors in the FA/BRCA pathway upregulated by melphalan in multidrug resistant cell line RPMI8226/R and indicate PJ34 can sensitize MDR cells with low concentration of melphalan, thus PJ34 can be used to treat refractory mutidrug resistant MM patients with low concentrations of melphalan.

Rad51 is the key recombination protein promoting the pairing and exchange of strands between homologous DNA molecules during HRR (homologous recombination repair) (44). Rad51 interacts with many accessory proteins in the FA/BRCA pathway and is involved in the repair of DNA cross-links $(9,10)$. Increased levels of Rad51 correlate with increased erroneous recombination and resistance to DNA-damaging agents in tumor cells (45). Rad51 overexpression leads to a worse clinical outcome in lung cancer (46). In the present study, PJ34 enhanced melphalan-induced cyto- toxicity via downregulation of Rad51 expression in human multidrug resistant RPMI8226/R cells. Our study has shown that the upregulation of Rad51 expression via the FA/BRCA pathway in melphalan-treated RPMI8226/R cells was required for cell survival and downregulation of Rad51 expression by PJ34 resulted in enhancement of melphalan sensitivity in resistant RPMI8226/R cells. However, a recent report showed that an MYC-dependent decrease in Rad51 expression is not sufficient to sensitize cells to MMC in H1299 lung cancer cells (47). Therefore, we suppose that Rad51 must be regulated together with other factors in the FA/BRCA pathway to maintain genomic stability in cells responding to DNA damage.

The sensitivity of cancer cells to chemotherapeutic druginduced DNA damage depends on the balance between DNA damage and repair. Therefore, targeting of DNA repair that promote cell survival is proposed as a promising strategy to enhance the efficacy of conventional chemotherapeutic agents. One signaling pathway that has recently drawn much attention for this purpose is the FA/BRCA pathway and abnormal activation of this pathway has been reported to play an important role in chemoresistance in a variety of tumors (11-23). These findings ignited enthusiasm for targeting DNA repair pathway as an anticancer modality and provide a superior strategy for overcoming development of resistance of cancer cells to targeted therapy. We found that PJ34 sensitized the multidrug resistant RPMI8226/R cells to melphalan and the basal levels of DSB repair proteins $\gamma$-H2AX increased when cells were treated with PJ34 which inhibited the FA/BRCA pathway activity. Thus, the possible mechanism is PJ34 might interfere with the DNA repair process by the suppression of the FA/BRCA pathway and cause damaged DNA to accumulate by slowing down the removal of DNA damage induced by melphalan, resulting in improved sensitivity to melphalan.

It has been reported that activation of the FA/BRCA pathway is supposed to be a resistance mechanism in alkylating agent-based chemotherapy (14-16) and PARP-1 inhibitors can improve the antitumor effect of alkylating agents (31-34). Thus, our findings suggest that concomitant targeting of the FA/BRCA pathway is a promising strategy to enhance antitumor effect of PJ34 in MM patients.

Although our present study has several limitations such as in vitro nature of the design and only one cell line tested, it demonstrated synergistic interaction between PJ34 and melphalan in multidrug resistant human MM cells. While PJ34 by itself showed only a limited antitumor effect, it synergistically potentiated melphalan-mediated apoptosis and DNA damage with activation of the FA/BRCA pathway in multidrug resistant RPMI8226/R cells. These findings suggest that targeting of DNA repair pathway can be a promising strategy for the patients with multidrug resistant multiple myeloma. Further comprehensive molecular studies should be performed to test the safety and in vivo synergistic antitumor effect of PJ34 and melphalan combination therapy for the clinical application in multidrug resistant multiple myeloma.

\section{Acknowledgements}

We thank Professor Jianfeng Zhou for providing the human multiple myeloma cell line RPMI8226. The present study was supported by the National Natural Science Foundation 
of China (grant no. 81001053) and the Fundamental Research Funds for the Central Universities (grant No. 4101041).

\section{References}

1. Spanswick VJ, Lowe HL, Newton C, et al: Evidence for different mechanisms of 'unhooking' for melphalan and cisplatin-induced DNA interstrand cross-links in vitro and in clinical acquired resistant tumour samples. BMC Cancer 12: 436, 2012.

2. Bouwman P and Jonker J: The effects of deregulated DNA damage signalling on cancer chemotherapy response and resistance. Nat Rev Cancer 12: 587-598, 2012.

3. Altieri F, Grillo C, Maceroni M, et al: DNA damage and repair: from molecular mechanisms to health implications. Antioxid Redox Signal 10: 891-937, 2008.

4. Hlavin EM, Smeaton MB and Miller PS: Initiation of DNA interstrand cross-link repair in mammalian cells. Environ Mol Mutagen 51: 604-624, 2010.

5. Bessho T: Induction of DNA replication-mediated double strand breaks by psoralen DNA interstrandcross-links. J Biol Chem 278: 5250-5254, 2003.

6. Sczepanski JT, Jacobs AC, Van Houten B, et al: Double-strand break formation during nucleotide excision repair of a DNA interstrand cross-link. Biochemistry 48: 7565-7567, 2009.

7. Kim H and D'Andrea AD: Regulation of DNA cross-link repair by the Fanconi anemia/BRCA pathway. Genes Dev 26 1393-1408, 2012.

8. Mouw KW and D'Andrea AD: Crosstalk between the nucleotide excision repair and Fanconi anemia/BRCA pathways. DNA Repair 19: 130-134, 2014.

9. Levitus M, Joenje H and de Winter JP: The Fanconi anemia pathway of genomic maintenance. Cell Oncol 28: 3-29, 2006.

10. Cohn MA and D'Andrea AD: Chromatin recruitment of DNA repair proteins: lessons from the fanconi anemia and doublestrand break repair pathways. Mol Cell 32: 306-312, 2008.

11. Rodríguez A, Sosa D, Torres L, et al: A Boolean network model of the FA/BRCA pathway. Bioinformatics 28: 858-866, 2012

12. Karanja KK, Cox SW, Duxin JP, et al: DNA2 and EXO1 in replication-coupled, homology-directed repair and in the interplay between HDR and the FA/BRCA network. Cell Cycle 11: 3983-3996, 2012.

13. Stecklein SR and Jensen RA: Identifying and exploiting defects in the Fanconi anemia/BRCA pathway in oncology. Transl Res 160: 178-197, 2012.

14. Jacquemont C, Simon JA, D'Andrea AD, et al: Non-specific chemical inhibition of the Fanconi anemia pathway sensitizes cancer cells to cisplatin. Mol Cancer 11: 26, 2012.

15. Chirnomas D, Taniguchi T, de la Vega M,etal: Chemosensitization to cisplatin by inhibitors of the Fanconi anemia/BRCA pathway. Mol Cancer Ther 5: 952-961, 2006.

16. Taniguchi T and D'Andrea AD: Molecular pathogenesis of Fanconi anemia: recent progress. Blood 107: 4223-4233, 2006.

17. Mori R, Yoshida K, Tanahashi T, et al: Decreased FANCJ caused by $5 \mathrm{FU}$ contributes to the increased sensitivity to oxaliplatin in gastric cancer cells. Gastric Cancer 16: 345-354, 2013.

18. Deans AJ and West SC: DNA interstrand crosslink repair and cancer. Nat Rev Cancer 11: 467-480, 2011.

19. Hegi ME, Sciuscio D, Murat A, et al: Epigenetic deregulation of DNA repair and its potential for therapy. Clin Cancer Res 15: 5026-5031, 2009.

20. Palagyi A, Neveling K, Plinninger U, et al: Genetic inactivation of the Fanconi anemia gene FANCC identified in the hepatocellular carcinoma cell line HuH-7 confers sensitivity towards DNA-interstrand crosslinking agents. Mol Cancer 9: 127, 2010.

21. Burkitt $\mathrm{K}$ and Ljungman M: Phenylbutyrate interferes with the Fanconi anemia and BRCA pathway and sensitizes head and neck cancer cells to cisplatin. Mol Cancer 7: 24, 2008.

22. Kondo N, Takahashi A, Mori E, et al: FANCD1/BRCA2 plays predominant role in the repair of DNA damage induced by ACNU or TMZ. PLoS One 6: e19659, 2011.

23. Yarde DN, Oliveira V, Mathews L, et al: Targeting the Fanconi anemia/BRCA pathway circumvents drug resistance in multiple myeloma. Cancer Res 69: 9367-9375, 2009.

24. Adhikari S, Choudhury S, Mitra PS, et al: Targeting base excision repair for chemosensitization. Anticancer Agents Med Chem 8: 351-357, 2008.
25. David KK, Andrabi SA, Dawson TM, et al: Parthanatos, a messenger of death. Front Biosci 14: 1116-1128, 2009.

26. Wang Y, Dawson VL and Dawson TM: Poly(ADP-ribose) signals to mitochondrial AIF: a key event in parthanatos. Exp Neurol 218: 193-202, 2009.

27. Bryant HE, Petermann E, Schultz N, et al: PARP is activated at stalled forks to mediate Mre11-dependent replication restart and recombination. EMBO J 28: 2601-2615, 2009.

28. Krishnakumar R and Kraus WL: The PARP side of the nucleus: molecular actions, physiological outcomes, and clinical targets. Mol Cell 39: 8-24, 2010.

29. Węsierska-Gądek J, Zulehner N, Ferk F, et al: PARP inhibition potentiates the cytotoxic activity of C-1305, a selective inhibitor of topoisomerase II, in human BRCA1-positive breast cancer cells. Biochem Pharmacol 84: 1318-1331, 2012.

30. Lavarone E, Puppin C, Passon N, et al: The PARP inhibitor PJ34 modifies proliferation, NIS expression and epigenetic marks in thyroid cancer cell lines. Mol Cell Endocrinol 365: 1-10, 2013.

31. Davidson D, Wang Y, Aloyz R, et al: The PARP inhibitor ABT-888 synergizes irinotecan treatment of colon cancer cell lines. Invest New Drugs 31: 461-468, 2013.

32. Cheng $\mathrm{H}$, Zhang Z, Borczuk A, et al: PARP inhibition selectively increases sensitivity to cisplatin in ERCC1-low non-small cell lung cancer cells. Carcinogenesis 34: 739-749, 2013.

33. Cavallo F, Graziani G, Antinozzi C, et al: Reduced proficiency in homologous recombination underlies the high sensitivity of embryonal carcinoma testicular germ cell tumors to cisplatin and poly (ADP-ribose) polymerase inhibition. PLoS One 7: e51563, 2012.

34. Donawho CK, Luo Y, Luo Y, et al: ABT-888, an orally active poly(ADP-ribose) polymerase inhibitor that potentiates DNA-damaging agents in preclinical tumor models. Clin Cancer Res 13: 2728-2737, 2007.

35. Ahel I, Ahel D, Matsusaka T, et al: Poly(ADP-ribose)-binding zinc finger motifs in DNA repair/checkpoint proteins. Nature 451: 81-85, 2008.

36. Pyriochou A, Olah G, Deitch EA, et al: Inhibition of angiogenesis by the poly(ADP-ribose) polymerase inhibitor PJ-34. Int J Mol Med 22: 113-118, 2008.

37. Xiao H, Xiao Q, Zhang K, et al: Reversal of multidrug resistance by curcumin through FA/BRCA pathway in multiple myeloma cell line MOLP-2/R. Ann Hematol 89: 399-404, 2010.

38. Vinod BS, Maliekal TT and Anto RJ: Phytochemicals as chemosensitizers: from molecular mechanism to clinical significance. Antioxid Redox Signal 18: 1307-1348, 2013.

39. Tomicic MT and Kaina B: Topoisomerase degradation, DSB repair, p53 and IAPs in cancer cell resistance to camptothecinlike topoisomerase I inhibitors. Biochim Biophys Acta 1835: $11-27,2013$

40. Liu X, Luo X, Shi Y, et al: Poly (ADP-ribose) polymerase activity regulates apoptosis in HeLa cells after alkylating DNA damage. Cancer Biol Ther 7: 934-941, 2008.

41. Gambi N, Tramontano F and Quesada P: Poly(ADPR) polymerase inhibition and apoptosis induction in cDDP-treated human carcinoma cell lines. Biochem Pharmacol 75: 2356-2363, 2008.

42. Yang YG, Cortes U, Patnaik S, et al: Ablation of PARP-1 does not interfere with the repair of DNA double-strand breaks, but compromises the reactivation of stalled replication forks. Oncogene 23: 3872-3882, 2004.

43. Mah LJ, Orlowski C, Ververis K, et al: Utility of $\gamma \mathrm{H} 2 \mathrm{AX}$ as a molecular marker of DNA double-strand breaks in nuclear medicine: applications to radionuclide therapy employing auger electron-emitting isotopes. Curr Radiopharm 4: 59-67, 2011.

44. Richardson C: RAD51, genomic stability, and tumorigenesis. Cancer Lett 218: 127-139, 2005.

45. Klein HL: The consequences of Rad51 overexpression for normal and tumor cells. DNA Repair 7: 686-693, 2008.

46. Qiao GB, Wu YL, Yang XN, et al: High-level expression of Rad51 is an independent prognostic marker of survival in nonsmall-cell lung cancer patients. Br J Cancer 93: 137-143, 2005.

47. Luoto KR, Meng AX, Wasylishen AR, et al: Tumor cell kill by c-MYC depletion: role of MYC-regulated genes that control DNA double-strand break repair. Cancer Res 70: 8748-8759, 2010. 